\title{
ADUBAÇÃO NITROGENADA E POTÁSSICA E SUA RELAÇÃO COM A INCIDÊNCIA DE CERCOSPORIOSE E FERRUGEM EM CAFEEIRO FERTIRRIGADO
}

\author{
Fabrício Júnio e Silva1', Gleice Aparecida Assis², Fabio Janoni Carvalho³, Bruno Sérgio \\ Vieira ${ }^{2}$, Laís Carvalho Santos ${ }^{3}$ \\ ${ }^{1}$ Faculdade Presidente Antônio Carlos de Uberlândia. Curso de Agronomia. \\ ${ }^{2}$ Programa de Pós-Graduação em Agricultura e Informações Geoespaciais, Universidade Federal de Uberlândia, Campus Monte \\ Carmelo. \\ ${ }^{3}$ Instituto Federal de Educação, Ciência e Tecnologia do Triângulo Mineiro, Uberaba. \\ *Autor para correspondência: Gleice Aparecida Assis, gleiceufu@gmail.com
}

RESUMO: O cafeeiro apresenta suscetibilidade a várias doenças, no entanto, as de maior importância e agressividade são a ferrugem, causada pelo fungo Hemileia vastatrix e a cercosporiose, cujo agente etiológico é o fungo Cercospora coffeicola. Objetivou-se avaliar a incidência de cercosporiose e ferrugem em cafeeiro fertirrigado com doses de nitrogênio e potássio na região de Monte Carmelo-MG. O trabalho foi conduzido na Fazenda Juliana, município de Monte Carmelo-MG. O plantio da lavoura foi realizado em novembro de 2011, utilizando-se mudas da cultivar Topázio MG 1190. Os tratamentos constaram de cinco níveis de $\mathrm{N}_{\text {e K}} \mathrm{O}$, correspondentes a 30, 80, 130, 180 e 230\% do recomendado para lavouras não irrigadas aplicados via fertirrigação. O parcelamento da adubação foi realizado em doze vezes iguais no ano, referente a uma fertirrigação por mês. O delineamento experimental utilizado foi em blocos casualizados, com quatro repetições. Cada parcela foi composta por 10 plantas, consideradas úteis as oito centrais. No período de março de 2015 a fevereiro de 2016, foi mensurado mensalmente a incidência da cercosporiose nas folhas e frutos e a incidência de ferrugem nas oito plantas úteis de cada parcela experimental. O nível de adubação de $30 \%$ do recomendado para lavouras cafeeiras favoreceu maior incidência de cercosporiose nos frutos. Os diferentes níveis de adubação não influenciaram na ocorrência da ferrugem ao longo do período avaliado.

PALAVRAS-CHAVE: Cercospora coffeicola, Coffea arabica L, fertirrigação, Hemileia vastatrix

\section{NITROGEN AND POTASSIUM FERTILIZATION AND ITS RELATIONSHIP WITH THE INCIDENCE OF CERCOSPORIOSIS AND RUST IN FERTIGATED COFFEE TREES}

\begin{abstract}
The coffee presents susceptibility to several diseases, however, those of greater importance and aggressiveness are rust, caused by the fungus Hemileia vastatrix and cercosporiose, whose etiological agent is the fungus Cercospora coffeicola. The aim of this study was to evaluate the occurrence of cercosporiosis and rust in fertirrigated coffee in the Monte Carmelo-MG. The work was carried out at Fazenda Juliana, Monte Carmelo-MG. The planting of the crop was carried out in November 2011, using seedlings of the cultivar Topázio. The treatments consisted of five levels of $\mathrm{N}$ and $\mathrm{K} 2 \mathrm{O}$, corresponding to 30 , $80,130,180$ and $230 \%$ of the recommended for non irrigated crops applied via fertirrigation. The fertilization was done in twelve times in the year, referring to one fertirrigation per month. The experimental design was in randomized blocks, with four replications. Each plot was composed of 10 plants, the eight plants considered useful. In the period from March 2015 to February 2016, the incidence of cercosporiosis in leaves and fruits and the incidence of rust on the eight useful plants of each experimental plot were measured monthly. The level of fertilization of $30 \%$ of the recommended for coffee plantations increased a incidence of cercosporiosis in the fruits. The different levels of fertilization did not influence the occurrence of rust during the evaluated period.
\end{abstract}

KEY WORDS: Cercospora coffeicola, Coffea arabica L, fertirrigation, Hemileia vastatrix

\section{INTRODUÇÃO}

O cafeeiro (Coffea arabica L.) é uma cultura de extrema relevância tanto para o abastecimento do mercado interno quanto para o externo. O Brasil é o maior produtor dessa commodity que gera renda para inúmeras pessoas que participam da cadeia produtiva desde o plantio até a sua comercialização.
O cafeeiro apresenta suscetibilidade a vários patógenos, uma das doenças de maior importância é a ferrugem, causada pelo fungo Hemileia vastatrix (Berk. e Broome), a qual incide sobre folhas, brotações novas e raramente em frutos. Os sintomas iniciais na face inferior das folhas são pequenas manchas amarelo-pálidas que se tornam amarelo-alaranjadas e pulverulentas, e quando 
coalescem cobrem grande parte do limbo foliar. Com o avanço da doença ocorre desfolha (Fazuoli et al., 2007). Sem a adoção de medidas de manejo adequadas pode reduzir a produtividade em 30 a 50\% (Tozzi e Ghini, 2016).

A cercosporiose, cujo agente etiológico é o fungo Cercospora coffeicola (Berk. e Cooke), em lavouras novas promove queda de folhas e frutos e seca de ramos produtivos e em lavouras adultas ocorre desfolha, amadurecimento precoce de frutos e chochamento (Carvalho et al., 2008), podendo ocasionar redução na produtividade em até 30\% (Souza et al., 2015).

Problemas de ordens nutricionais desencadeiam nos vegetais um aumento na predisposição do hospedeiro a vários patógenos pela diminuição à tolerância ou resistência aos mesmos (Pozza et al., 2001). O manejo da adubação deve considerar alguns fatores como exigência nutricional da planta, a fertilidade natural do solo, o sistema de cultivo, o estado nutricional da lavoura, a produtividade esperada para a próxima safra, entre outros. A deficiência ou o excesso de nutrientes prontamente disponíveis para as plantas podem acarretar perdas de produção (Pozza et al., 2001).

O nitrogênio contribui para a síntese de lignina, fitoalexinas e taninos, em excesso, reduz a produção desses compostos, devido à demanda de carbono na fotossíntese via ciclo de Krebs, comprometendo a síntese dos metabólitos secundários pela via do ácido chiquímico e contribui para a liberação de polissacarídeos na superfície foliar. A adubação nitrogenada e sua relação à resistência das plantas às doenças variam em função do patógeno, genótipo, dose e fonte do nutriente utilizado e da interação entre nutrientes. Altos teores de $\mathrm{N}$ promovem aumento na produção de tecidos jovens e suculentos. O aumento das doses de $\mathrm{N}$ pode promover uma redução de $20,7 \%$ na área abaixo da curva de progresso do número de lesões de C. coffeicola por folha (Pozza et al., 2001).

O potássio confere maior resistência aos tecidos das plantas por aumentar a espessura da cutícula e da parede celular. Lima et al. (2010) verificaram que houve uma redução da intensidade da mancha de Phoma (agente etiológico Phoma tarda) com o aumento das doses de $\mathrm{K}$ até a dose de $7 \mathrm{mmol} \mathrm{L} \mathrm{L}^{-1}$ em cafeeiros cultivados em solução nutritiva, o que reforça a função deste nutriente em conferir maior dificuldade à penetração dos patógenos e consequentemente ao processo de infecção.

A irrigação como fator isolado não determina a incidência de doenças, porém proporciona microclima favorável ao patógeno. A falta de umidade no solo pode condicionar à planta menor absorção de nutrientes, tornando-a suscetível à infecção de C. coffeicola. A fertirrigação disponibiliza água e nutrientes para às raízes e não promove molhamento foliar, não favorecendo a disseminação e a germinação dos uredósporos de $H$. vastatrix. Já em áreas irrigadas com pivô central a doença apresenta comportamento cíclico (Talamini et al., 2003).

Ciente da importância da nutrição da planta e das condições edafoclimáticas para a ocorrência de doenças, objetivou-se com este trabalho avaliar a incidência de cercosporiose e ferrugem em cafeeiro fertirrigado com doses de nitrogênio e potássio na região de Monte Carmelo-MG.

\section{MATERIAL E MÉTODOS}

O experimento foi conduzido na Fazenda Juliana, no município de Monte Carmelo localizado na mesorregião do Triângulo Mineiro e Alto Paranaíba situado a uma altitude de $911 \mathrm{~m}$, latitude sul de $18^{\circ} 42^{\prime} 31^{\prime \prime}$ e longitude oeste de $47^{\circ} 32^{\prime} 47^{\prime \prime}$. O plantio da lavoura foi realizado em novembro de 2011, utilizando-se mudas da cultivar Topázio MG 1190. Foi adotado espaçamento de $3,8 \mathrm{~m}$ entre linhas por $0,6 \mathrm{~m}$ entre plantas, totalizando 4.386 plantas ha- ${ }^{-1}$, considerado um sistema de plantio semi-adensado. Os dados meteorológicos de março de 2015 a fevereiro de 2016, referente ao período de avaliação das doenças no campo, foram obtidos na estação climatológica da Fazenda Juliana (Figura 1). O solo foi analisado quanto às características químicas na camada de 0 a $20 \mathrm{~cm}$, no período de 2012 a 2015 (Tabela 1).

Foram utilizados cinco níveis de $\mathrm{N}$ e $\mathrm{K}_{2} \mathrm{O}$ (30\%, $80 \%, 130 \%, 180 \%$ e $230 \%$ do recomendado para lavouras não irrigadas) aplicados via fertirrigação, segundo Guimarães et al. (1999). A adubação foi parcelada em 12 vezes iguais ao longo do ano, referente a uma fertirrigação por mês. $\mathrm{Na}$ adubação das safras 2014/2015 e 2015/2016 foram utilizados os adubos ureia pecuária $(45 \%$ de $\mathrm{N})$ e nitrato de potássio ( $44 \%$ de $\mathrm{K}_{2} \mathrm{O}$ e $13 \%$ de $\mathrm{N}$ ), fontes solúveis indicadas para a fertirrigação (Tabela 2).

$O$ delineamento experimental utilizado foi o de blocos casualizados, com quatro repetições. Cada parcela foi composta por 10 plantas, sendo úteis para avaliação as oito plantas centrais. Cada linha de plantio foi intercalada com duas linhas de bordadura, de forma a evitar uma possível interferência de um tratamento de fertirrigação sobre o outro. $\mathrm{O}$ experimento apresentou 20 parcelas. 
Figura 1. Dados meteorológicos da área experimental no período de março de 2015 a fevereiro de 2016.

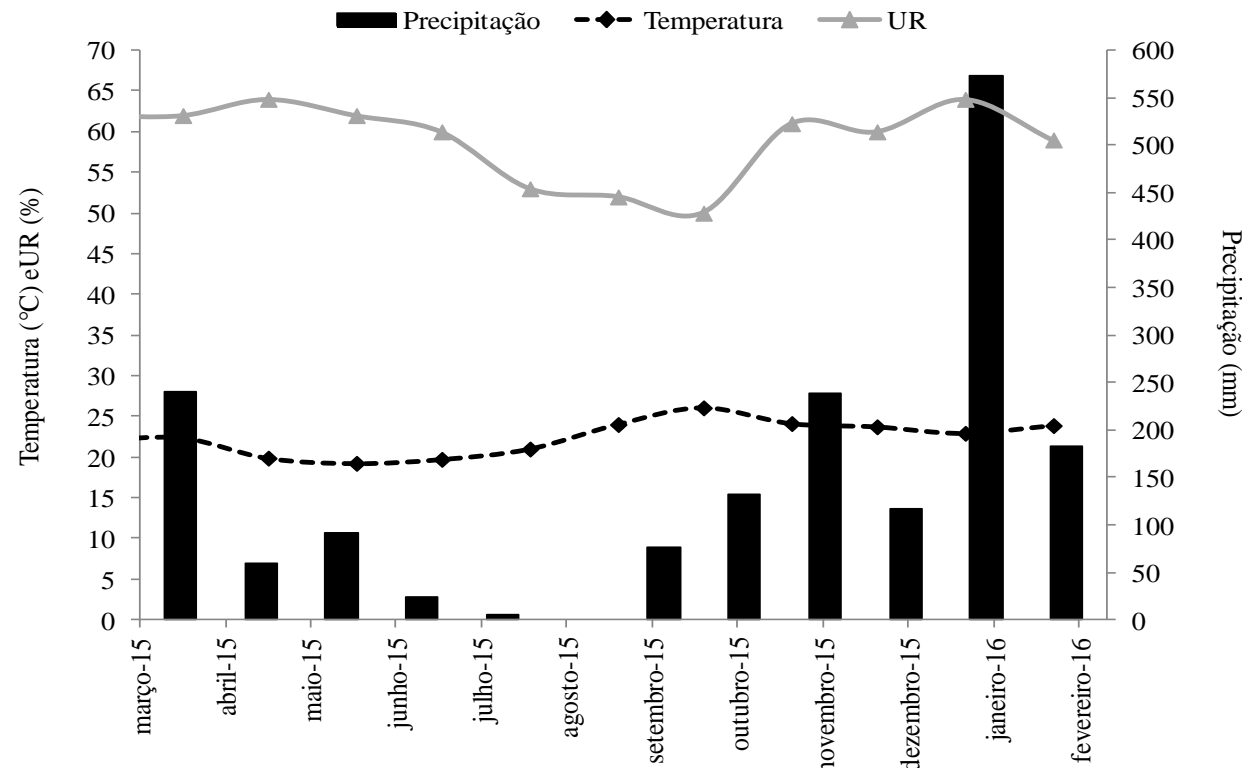

Tabela 1. Caracterização química do solo na camada de 0-20 cm em 2012, 2013, 2014 e 2015.

\begin{tabular}{|c|c|c|c|c|c|}
\hline \multicolumn{2}{|l|}{ Característica } & 2012 & 2013 & 2014 & 2015 \\
\hline \multicolumn{2}{|l|}{$\mathrm{pH}\left(\mathrm{H}_{2} \mathrm{O}\right)$} & 6,1 & 6,3 & 5,5 & 6,8 \\
\hline \multicolumn{2}{|l|}{ Fósforo $(P)-\mathrm{mg} \mathrm{dm}^{-3}$} & 10,5 & 62,4 & 4,4 & 7,4 \\
\hline \multicolumn{2}{|l|}{ Potássio $(\mathrm{K})$ - mg dm -3 } & 69,0 & 222,0 & 74,0 & 110,0 \\
\hline \multicolumn{2}{|c|}{ Cálcio $\left(\mathrm{Ca}^{2+}\right)-$ cmolc dm $^{-3}$} & 2,6 & 6,0 & 1,4 & 3,3 \\
\hline \multicolumn{2}{|c|}{ Magnésio $\left(\mathrm{Mg}^{2+}\right)-$ cmolc dm ${ }^{-3}$} & 1,8 & 1,1 & 0,5 & 1,6 \\
\hline \multicolumn{2}{|c|}{ 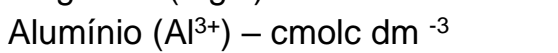 } & 0,55 & 0,0 & 0,0 & 0,0 \\
\hline \multicolumn{2}{|c|}{$\mathrm{H}+\mathrm{Al}$ (Extrator SMP) - cmolc $\mathrm{dm}^{-3}$} & 2,5 & 1,8 & 3,3 & 1,9 \\
\hline \multicolumn{2}{|c|}{$\mathrm{SB}-\mathrm{cmolc} \mathrm{dm}^{-3}$} & 4,5 & 7,6 & 2,0 & 5,2 \\
\hline \multicolumn{2}{|l|}{ CTC $(\mathrm{t})-\mathrm{cmolc} \mathrm{dm}^{-3}$} & 5,1 & 7,6 & 2,1 & 5,2 \\
\hline \multicolumn{2}{|c|}{ CTC a pH 7,0 (T) - cmolc dm ${ }^{-3}$} & 7,0 & 9,4 & 5,3 & 7,1 \\
\hline \multicolumn{2}{|c|}{ Saturação por bases (V) - \% } & 65,0 & 81,0 & 39,0 & 73,0 \\
\hline \multicolumn{2}{|c|}{ Saturação de alumínio (m) - \% } & 11,0 & 0,0 & 3,0 & 0,0 \\
\hline \multicolumn{2}{|c|}{ Matéria orgânica (MO) - dag kg-1 } & 2,3 & 3,4 & 1,8 & 2,4 \\
\hline \multicolumn{2}{|c|}{ Zinco $(Z n)-\mathrm{mg} \mathrm{dm}^{-3}$} & 1,9 & 3,8 & 1,0 & 1,6 \\
\hline \multicolumn{2}{|l|}{ Ferro $(\mathrm{Fe})-\mathrm{mg} \mathrm{dm}^{-3}$} & 41,0 & 31,0 & 49,0 & 48,0 \\
\hline \multicolumn{2}{|c|}{ Manganês (Mn) $-\mathrm{mg} \mathrm{dm}^{-3}$} & 5,4 & 8,9 & 3,2 & 6,0 \\
\hline \multicolumn{2}{|c|}{ Cobre $(\mathrm{Cu})-\mathrm{mg} \mathrm{dm}^{-3}$} & 0,2 & 3,8 & 0,3 & 0,3 \\
\hline \multicolumn{2}{|l|}{ Boro (B) $-\mathrm{mg} \mathrm{dm}^{-3}$} & 0,14 & 0,5 & 0,3 & 0,3 \\
\hline \multicolumn{2}{|l|}{ Enxofre $(S)-\mathrm{mg} \mathrm{dm}^{-3}$} & 23,0 & 216,0 & 5,0 & - \\
\hline \multicolumn{6}{|c|}{ 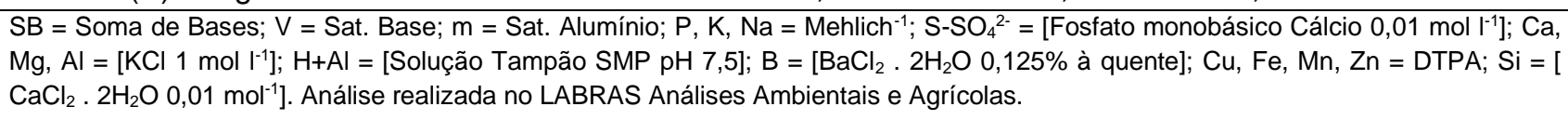 } \\
\hline \multirow{2}{*}{ Níveis de adubação* } & \multicolumn{2}{|c|}{$2014 / 2015$} & \multicolumn{3}{|c|}{$2015 / 2016$} \\
\hline & $\mathrm{N}$ & $\mathrm{K}_{2} \mathrm{O}$ & $\mathrm{N}$ & & $\mathrm{K}_{2} \mathrm{O}$ \\
\hline $30 \%$ & 88,5 & 67,5 & 117,8 & & 90,0 \\
\hline $80 \%$ & 235,9 & 180,0 & 314,2 & & 239,9 \\
\hline $130 \%$ & 383,4 & 292,5 & 510,6 & & 390,0 \\
\hline $180 \%$ & 530,8 & 405,0 & 707,0 & & 540,0 \\
\hline $230 \%$ & 678,3 & 517,5 & 903,5 & & 690,0 \\
\hline
\end{tabular}

* Níveis de adubação baseados na recomendação de Guimarães et al. (1999).

O sistema de irrigação automatizado constou de uma unidade central de controle (motobomba, filtros de disco, manômetros e conexões), linha principal de tubos PVC, PN 80, linhas de derivação, registros e controle de válvulas automatizado com controlador modelo NMC-PRO Netafim. Para 
irrigação, foi utilizado tubo gotejador autocompensante de parede grossa $(0,9 \mathrm{~mm})$, com emissores com vazão de $2,2 \mathrm{~L} \mathrm{~h}^{-1}$, espaçados a cada $0,6 \mathrm{~m}$.

A cada 30 dias foram avaliados a incidência de cercosporiose nas folhas e frutos e a de ferrugem em oito plantas de cada parcela experimental, no período de 01 de março de 2015 a 01 de fevereiro de 2016, totalizando 12 avaliações. Para avaliação da ferrugem foi utilizado o método não destrutivo, consistindo no monitoramento aleatório do terceiro ou quarto par de folhas do ramo plagiotrópico localizada no terço inferior da planta. No caso da cercosporiose, foi avaliado o $3^{\circ}$ ou $4^{\circ}$ par de folhas do ramo plagiotrópico localizado no terço médio da planta. A cercosporiose também foi avaliada nos frutos no estádio cereja no mês de junho de 2015, sendo os mesmos coletados no terço médio das plantas de cada unidade experimental, avaliadas por incidência, estimados pela percentagem de frutos lesionados (Santos et al., 2008).

Para os meses com presença de cercosporiose ou ferrugem nas folhas, os dados com distribuição Binomial e função de ligação logit foram analisados utilizando o Modelo Linear Generalizado, correspondendo as oito plantas amostradas ao número de tentativas e a presença da doença ao evento de sucesso. Foi detectada diferença entre os tratamentos quando os desvios provocados por este fator (deviance) foram significativos pelo teste de Qui-Quadrado a 0,05 de significância. Quando significativos, as médias dos tratamentos foram comparadas pelo teste de $t$ de Student com ajuste de Bonferroni, a 0,05 de significância. $O$ mesmo procedimento foi adotado para a presença da cercosporiose nos frutos ao final do experimento.

\section{RESULTADOS E DISCUSSÃO}

Os níveis de adubação nitrogenada e potássica afetaram a incidência de cercosporiose nos frutos de cafeeiro de acordo com a análise de deviance. O nível de adubação de $30 \%$ do recomendado para lavouras cafeeiras de sequeiro diferenciou-se dos demais tratamentos, favorecendo maior incidência da doença nos frutos, em média, $3,59 \%$ superior em relação aos outros tratamentos (Tabela 3). Um dos possíveis fatores que pode ter contribuído para esse resultado é a deficiência de potássio nos cafeeiros que receberam adubação com base apenas em $30 \%$ do que seria recomendado para uma lavoura não irrigada. Enquanto que nesse nível de adubação as plantas foram adubadas com $117,8 \mathrm{Kg}^{-1} \mathrm{ano}^{-1}$ de $\mathrm{N}$ e $90,0 \mathrm{Kg}$ ha $^{-1}$ ano $^{-1}$ de $\mathrm{K}_{2} \mathrm{O}$ (Tabela 2), na recomendação de Guimarães et al. (1999), esses valores seriam de $392,6 \mathrm{Kg} \mathrm{ha}^{-1} \mathrm{ano}^{-1}$ de $\mathrm{N}$ e $300 \mathrm{Kg}$ ha $^{-1}$ ano $^{-1}$ de $\mathrm{K}_{2} \mathrm{O}$. O nível de adubação de $30 \%$ da recomendação é insuficiente para a planta em termos de resistência à patógenos, trazendo um desequilíbrio nutricional e consequentemente favorecendo a incidência da doença.

Tabela 3. Incidência de cercosporiose nos frutos de cafeeiros sob diferentes níveis de nitrogênio e potássio na fertirrigação.

\begin{tabular}{|c|c|}
\hline $\begin{array}{c}\text { Níveis de adubação } \\
(\%)\end{array}$ & $\begin{array}{c}\text { Cercosporiose nos frutos } \\
(\%)\end{array}$ \\
\hline 30 & $6,07 \mathrm{~b}$ \\
\hline 80 & $1,72 \mathrm{a}$ \\
\hline 130 & $2,83 \mathrm{a}$ \\
\hline 180 & $2,95 \mathrm{a}$ \\
\hline 230 & $2,41 a$ \\
\hline
\end{tabular}

significativamente entre si pelo teste $t$ de Student ao nível de $5 \%$ de probabilidade.

Vários autores já relataram em suas pesquisas a importância de uma adubação equilibrada e sua relação com a sanidade vegetal. De acordo com Santos et al. (2008), a cercosporiose é favorecida pelo desequilíbrio nutricional, principalmente entre o cálcio e potássio provocado por safras de grande carga pendente juntamente com a ausência de chuvas ou ainda por excesso de adubação de cobertura. O potássio é responsável pela resistência do vegetal às doenças, pela recuperação de tecidos danificados, aumentando a espessura da parede celular e da cutícula. Porém, o excesso de potássio inibe a absorção de $\mathrm{Ca}$ e $\mathrm{Mg}$ pela planta, os quais competem pelo sítio de absorção do K, favorecendo assim a cercosporiose. A bienalidade também é um fator que influencia no desequilíbrio nutricional da planta. A maior ou menor relação C:N (20:1 a 25:1) pode causar a imobilização ou até mesmo a lixiviação de nutrientes, sendo importante fornecer fertilizantes que proporcionem de forma ideal a sincronização entre a liberação dos nutrientes e a demanda do cafeeiro (Patricio e Braghini, 2011).

Para a incidência de cercosporiose nas folhas, verificou-se diferença significativa entre os tratamentos de acordo com análise de deviance nos meses de março, maio e julho. O clima apresenta grande influência na ocorrência e progresso dessa doença, em especial temperatura e precipitação. A Figura 2 representa a incidência de cercosporiose no cafeeiro nas diferentes épocas do ano em função das variáveis meteorológicas no município de Monte Carmelo-MG. 
Figura 2. Porcentagem de folhas com cercosporiose em relação à temperatura média e precipitação em diferentes épocas do ano.

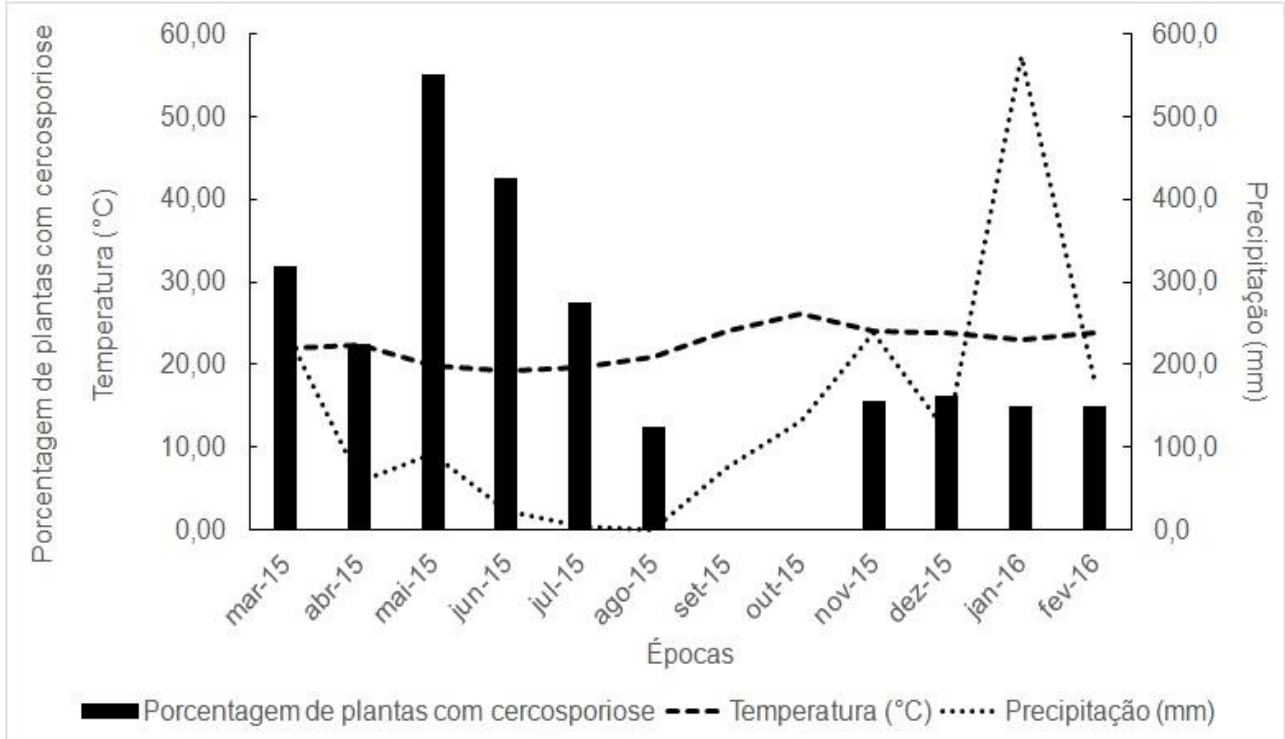

Pode-se notar a incidência da doença em quase todos os meses do ano (exceto em setembro e outubro), com maior porcentagem de plantas lesionadas nos meses de maio, junho e julho quando comparado às demais épocas, o que pode estar relacionado às baixas precipitações ocorridas no período, favorecendo a ocorrência de cercosporiose. De acordo com Salgado et al. (2007), o déficit hídrico é um dos fatores que colaboram para a incidência da doença, além de temperaturas entre $24^{\circ} \mathrm{C}$ e $30^{\circ} \mathrm{C}$ que favorecem a germinação dos conídios e crescimento do fungo.

Lopes et. al. (2012) também constataram em cafeeiro cultivado em sistema convencional no município de Poço Fundo, Sul de Minas Gerais, maior índice de ataque da cercosporiose no mês de julho (39\%), coincidindo com um ano de alta carga de frutos pendentes e muita insolação sobre os cafeeiros. Nesta lavoura a doença teve decréscimo a partir de setembro, o que corrobora as informações obtidas neste experimento.

Logo após a colheita, realizada em julho de 2015, notou-se uma renovação das folhas de todos os cafeeiros das parcelas experimentais. As plantas ficaram com aspecto mais vigoroso e as folhas velhas com sintomas da doença caíram, o que explica o decréscimo da doença após esse período (Cunha et al., 2004).

Os diferentes níveis de $\mathrm{N}$ e $\mathrm{K}_{2} \mathrm{O}$ não afetaram a ferrugem no cafeeiro, com exceção no mês de maio, de acordo com a análise de deviance (Tabela 4). Nota-se ausência da doença entre os meses de agosto de 2015 a fevereiro de 2016 e maior porcentagem de ocorrência de plantas com ferrugem entre os meses de março a julho com pico no mês de maio (17,5\%) (Figura 3 ) devido a baixas temperaturas $\left(19,9^{\circ} \mathrm{C}\right)$ e altas precipitações (92 $\mathrm{mm})$, seguido do mês de junho $(12,5 \%)$ com temperatura de $29,26^{\circ} \mathrm{C}$ e precipitações de 23,6 $\mathrm{mm}$, março $(7,5 \%)$ com temperatura de $22,7^{\circ} \mathrm{C}$ e precipitações de $240,8 \mathrm{~mm}$ e posteriormente dos meses de abril e julho (ambos com 4,8\%) (Figura 1). A mesma tendência foi observada em lavoura cafeeira fertirrigada em Lavras, Sul de Minas Gerais, onde o pico da ferrugem ocorreu em maio, provavelmente devido às temperaturas favoráveis ao patógeno responsável pela doença (Paiva et al., 2011).

De acordo com Chalfoun et al. (2005) ao longo dos anos houve uma alteração na curva de progresso da ferrugem devido ao atraso do início da estação chuvosa e temperaturas elevadas no início do verão, condições estas desfavoráveis ao ciclo de vida do patógeno (ferrugem tardia). Isso promoveu uma inflexão da curva de progresso da ferrugem mudando os meses de incidência para épocas tardias, quando comparado com a curva normal.

No presente trabalho, a temperatura média de $20,6^{\circ} \mathrm{C}$ entre os meses de maio a julho (Figura 1) favoreceu a germinação dos uredósporos de $H$. vastatrix. Paulo et al. (2013) constataram que temperaturas médias entre $15^{\circ} \mathrm{C}$ e $28,5^{\circ} \mathrm{C}$ são condições térmicas que não causam a inibição da germinação dos uredósporos do fungo, ao contrário de temperaturas altas entre $34^{\circ} \mathrm{C}$ a $38^{\circ} \mathrm{C}$ que impedem o desenvolvimento normal dos esporos. Tal fato não ocorreu em nenhum dos meses avaliados, pois a temperatura não ultrapassou $30^{\circ} \mathrm{C}$. 
Tabela 4. Análise de Deviance para incidência de ferrugem nas folhas de cafeeiros em função dos níveis de adubação nitrogenada em diferentes épocas do ano para uma distribuição binominal e função de ligação logit.

\begin{tabular}{|c|c|c|c|c|}
\hline \multicolumn{5}{|c|}{ Março } \\
\hline Modelo & GL & Deviance & $x^{2}$ & Prob. \\
\hline Nulo & 0 & & 29,77 & \\
\hline Tratamento & 4 & 7,69 & 22,08 & 0,103 \\
\hline Bloco & 3 & 5,79 & 16,29 & 0,12 \\
\hline \multicolumn{5}{|c|}{ Abril } \\
\hline Modelo & $\mathrm{GL}$ & Deviance & $x^{2}$ & Prob. \\
\hline Nulo & 0 & & 15,30 & \\
\hline Tratamento & 4 & 3,71 & 11,58 & 0,446 \\
\hline Bloco & 3 & 4,46 & 7,12 & 0,22 \\
\hline \multicolumn{5}{|c|}{ Maio } \\
\hline Modelo & GL & Deviance & $x^{2}$ & Prob. \\
\hline Nulo & 0 & & 55,18 & \\
\hline Tratamento & 4 & 10,84 & 44,35 & 0,028 \\
\hline Bloco & 3 & 15,06 & 29,29 & 0,00 \\
\hline \multicolumn{5}{|c|}{ Junho } \\
\hline Modelo & GL & Deviance & $x^{2}$ & Prob. \\
\hline Nulo & 0 & & 32,51 & \\
\hline Tratamento & 4 & 7,81 & 24,69 & 0,090 \\
\hline Bloco & 3 & 7,59 & 17,10 & 0,06 \\
\hline \multicolumn{5}{|c|}{ Julho } \\
\hline Modelo & $\overline{G L}$ & Deviance & $x^{2}$ & Prob. \\
\hline Nulo & 0 & & 18,36 & \\
\hline Tratamento & 4 & 1,10 & 16,37 & 0,738 \\
\hline Bloco & 3 & 0,51 & 15,86 & 0,92 \\
\hline
\end{tabular}

${ }^{*} \mathrm{GL}=$ grau de liberdade

Figura 3. Porcentagem de folhas com ferrugem em relação à temperatura média e precipitação em diferentes épocas do ano.

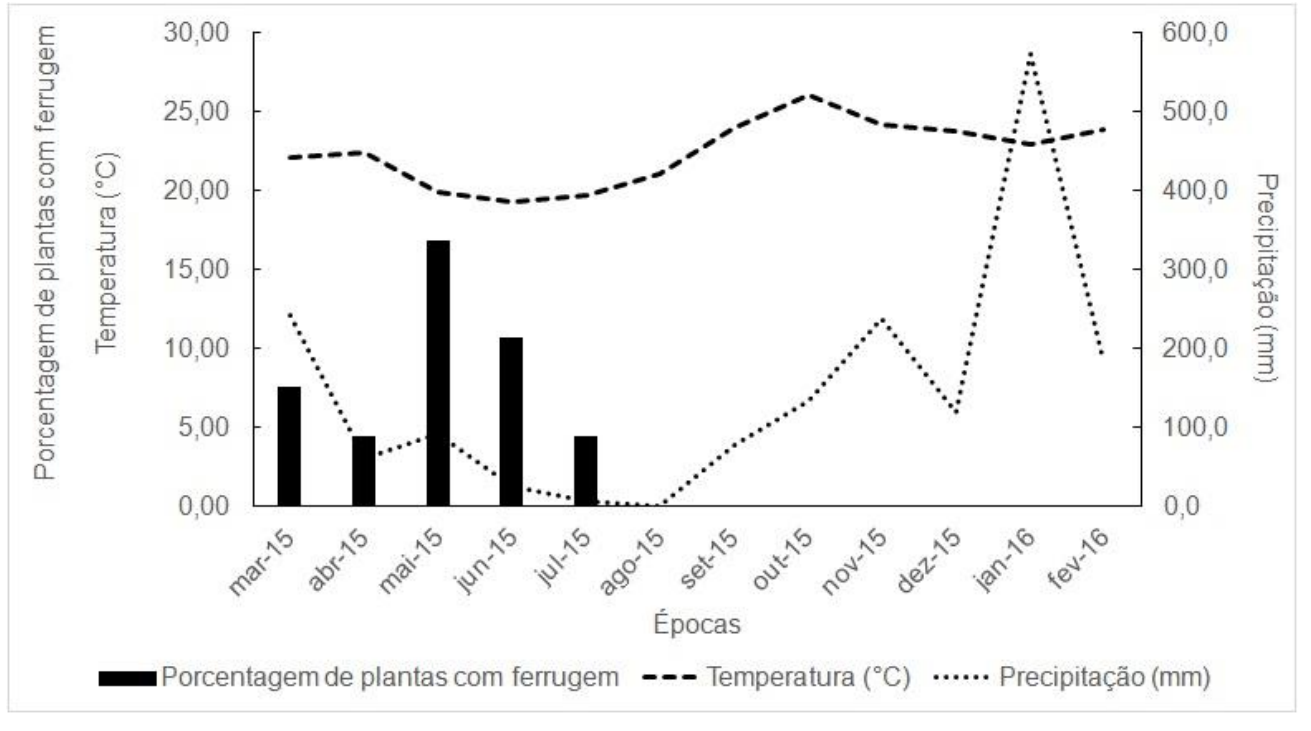

A precipitação pluviométrica nos meses de maior ocorrência de ferrugem $(420,2 \mathrm{~mm})$ foi inferior às demais épocas avaliadas $(1.320,4 \mathrm{~mm})$. A incidência da ferrugem não se correlaciona diretamente com o aumento ou diminuição da precipitação pluviométrica de forma isolada, sem considerar outros fatores, o que foi constatado por Paulo et al. (2013).

Paiva et al. (2011) concluíram que plantios adensados (5.000 a 10.000 plantas $^{-1}{ }^{-1}$ ) favorecem significativamente a progressão da ferrugem em maior proporção (84\%) em relação ao plantio convencional (2.500 plantas ha- $\left.{ }^{-1}\right)$, o qual ficou 
abaixo da curva de progresso da doença (52\%). A área experimental deste estudo enquadra-se no plantio semi-adensado (4.386 plantas ha-1) podendo assim ter contribuído de forma indireta para a doença, proporcionando um microclima favorável para o desenvolvimento do patógeno.

Com base no estudo realizado, verifica-se que o nível de adubação de $30 \%$ do recomendado para lavouras cafeeiras de sequeiro favorece maior incidência de cercosporiose nos frutos. Além disso, os diferentes níveis de adubação não influenciaram na incidência da ferrugem ao longo do período avaliado.

\section{REFERÊNCIAS BIBLIOGRÁFICAS}

Carvalho, V. L. de., Cunha, R. L. da; Moura, P. H. A. Manejo Integrado da Cercosporiose do Cafeeiro. Belo Horizonte: EPAMIG, 2008, 1-4 (EPAMIG, Circular Técnica, 16).

Chalfoun, S. M.; Souza-Pimentel, G. C. de.; Carvalho, V. L. de. Ferrugem-do-cafeeiro: importância do monitoramento com as mudanças no clima. Belo Horizonte: EPAMIG, 2005, 1-5 (EPAMIG, Circular Técnica, 235).

Cunha, R. L. da; Mendes, A. N. G.; Chalfoun, S. M. Controle químico da ferrugem do cafeeiro (Coffea arabica L.) e seus efeitos na produção e preservação do enfolhamento. Ciência e Agrotecnologia, 2004, 5, 28, 990-996.

Fazuoli, L. C.; Braghini, M. T.; Silvarolla, M. B.; Oliveira, A. C. B. de. A ferrugem alaranjada do cafeeiro e a obtenção de cultivares resistentes. $O$ Agronômico, 2007, 59, 48-53.

Guimarães, P. T. G.; Ribeiro, A. C.; Alvarez, V.; Vitor, $H$. Recomendações para o uso de corretivos e fertilizantes em Minas Gerais: 5ำ aproximação. Viçosa, MG: CFSEMG, 1999, 289-302.

Lima, L. M. de; Pozza, E. A.; Torres, H. N; Pozza, A. A. A.; Salgado, M.; Pfenning, L. H. Relação nitrogênio/potássio com mancha de Phoma e nutrição de mudas de cafeeiro em solução nutritiva. Tropical Plant Pathology, 2010, 35, 4, 223-228.

Lopes, P. R.; Ferraz, J. M. G.; Theodoro, V. C. de A.; Lopes, I. M. Evolução da ferrugem (Hemileia vastatrix) e da cercosporiose (Cercospora coffeicola) em agroecossistemas cafeeiros convencional, organo-mineral e orgânico. Revista Brasileira de Agroecologia, 2012, 7, 1, 1-9.

Paiva, B. R. T. L.; Souza, P. E. de; Scalco, M. S.; Santos, L. A. Progresso da ferrugem do cafeeiro irrigado em diferentes densidades de plantio póspoda. Ciência e Agrotecnologia, 2011, 35, 1, 1-7.

Paulo, E. M.; Montes, S. M. N. M.; Fischer, I. H. Progresso temporal da ferrugem alaranjada em cultivares de cafeeiro no oeste de São Paulo. Arquivo do Instituto Biológico, 2013, 80, 1, 1-6.

Patrício, F. R. A.; Braghini, M.T. Efeito de fungicidas triazóis sobre o controle da cercosporiose em mudas de cafeeiro. Arquivo do Instituto Biológico, 2011, 78, 2, 1-9.

Pozza, A. A. A.; Prieto Martinez, H. E.; Caixeta, S. L.; Cardoso, A. A.; Zambolim, L.; Pozza, E. A. Influência da nutrição mineral na intensidade da mancha-de-olho-pardo em mudas de cafeeiro. Pesquisa Agropecuária Brasileira, 2001, 36, 1, 5360.

Salgado, B. G.; Macedo, R. L. G.; Carvalho, V. L. de; Salgado, M.; Venturin, N. Progresso da ferrugem e da cercosporiose do cafeeiro consorciado com grevílea, com ingazeiro e a pleno sol em Lavras MG. Ciência e Agrotecnologia, 2007, 31, 4, 1-8.

Santos, F. da S.; Souza, P. E. de; Pozza, E. A.; Miranda, J. C.; Barreto, S. S.; Theodoro, V. C. Progresso da cercosporiose (Cercospora coffeicola Berkeley \& Cooke) em cafeeiros sob cultivos orgânico e convencional. Summa Phytopathologica, 2008, 34, 1, 1-7.

Santos, F. da S.; Souza, P. E. de.; Pozza, E. A.; Miranda, J. C.; Carvalho, E. A.; Fernandes, L. H. M.; Pozza, A. A. A. Adubação orgânica, nutrição e progresso de cercosporiose e ferrugem-do-cafeeiro. Pesquisa Agropecuária Brasileira, 2008, 43, 7, 783791.

Souza, A. G. C.; Maffia, L. A.; Silva, F. F.; Mizubuti, E. S. G.; Teixeira, H. A time series analysis of brown eye spot progress in conventional and organic coffee production systems. Plant Pathology, 2015, 64, 157-166.

Talamini, V.; Pozza, E. A.; Souza, P. E. de.; Silva, A. M. da. Progresso da ferrugem e da cercosporiose em cafeeiro (Coffea arabica I.) com diferentes épocas de início e parcelamentos da fertirrigação. Ciência e Agrotecnologia, 2003, 27, 1, 141-149.

Tozzi, F. R. O.; Ghini, R. Impacto do aumento da concentração atmosférica de dióxido de carbono sobre a ferrugem e o crescimento do cafeeiro. Pesquisa Agropecuária Brasileira, 2016, 51, 8, 933941. 